\title{
Frontières
}

\section{Travaux de la Commission fédérale belge de contrôle et d'évaluation de l'application de la loi du 28 mai 2002 relative à l'euthanasie}

\section{Fernand Van Neste}

Volume 24, numéro 1-2, automne 2011, printemps 2012

L’aide médicale à mourir

URI : https://id.erudit.org/iderudit/1013088ar

DOI : https://doi.org/10.7202/1013088ar

Aller au sommaire du numéro

Éditeur(s)

Université du Québec à Montréal

ISSN

1916-0976 (numérique)

Découvrir la revue

Citer cet article

Van Neste, F. (2011). Travaux de la Commission fédérale belge de contrôle et d'évaluation de l'application de la loi du 28 mai 2002 relative à l'euthanasie. Frontières, 24(1-2), 81-87. https://doi.org/10.7202/1013088ar

\section{Résumé de l'article}

La loi relative à l'euthanasie prévoit qu'une commission soit établie afin de contrôler et d'évaluer très attentivement son application. Ce contrôle se fait par un document d'enregistrement que tout médecin, ayant pratiqué une euthanasie, doit remplir et envoyer à la commission. Celle-ci assure ainsi un suivi permanent de l'application de la loi. L'article analyse les travaux de la commission. Il en ressort que, par ces travaux, le législateur est bien informé de l'application de la loi. De plus, ce contrôle et cette évaluation constituent une méthode efficace et constructive pour aider les médecins à agir correctement à l'égard des malades en fin de vie. 
Résumé

La loi relative à l'euthanasie prévoit qu'une commission soit établie afin de contrôler et d'évaluer très attentivement son application. Ce contrôle se fait par un document d'enregistrement que tout médecin, ayant pratiqué une euthanasie, doit remplir et envoyer à la commission. Celle-ci assure ainsi un suivi permanent de l'application de la loi. L'article analyse les travaux de la commission. Il en ressort que, par ces travaux, le législateur est bien informé de l'application de la loi. De plus, ce contrôle et cette évaluation constituent une méthode efficace et constructive pour aider les médecins à agir correctement à l'égard des malades en fin de vie.

Mots clés: contrôle et évaluation de l'application de la loi interprétation des conditions légales requises pour pratiquer l'euthanasie euthanasie active - euthanasie passive.

\section{Abstract}

The law on euthanasia provides that a commission be established to supervise and evaluate the implementation of the law concerned. This supervision is realised by means of a registration document which each physician having practiced euthanasia must fill in and send to the commission. In this way, the commission assures a permanent follow-up of the implementation of the law.

The article analyzes the activities of the commission. It reveals that, by these activities, the legislator is well-informed about the implementation of the law. Moreover, this supervision and evaluation constitute an efficient and constructive method to help the physicians act correctly, with regard to the sick persons at the end of their live.

Key words: supervision and evaluation of the implementation of the law interpretation of the legal conditions required for practicing euthanasia active euthanasia, passive euthanasia.

\section{Travaux de la Commission fédérale belge de contrôle et d'évaluation de l'application de la loi du 28 mai 2002 relative à l'euthanasie}

Fernand Van Neste, s. J., professeur émérite de l'Université d'Anvers,

docteur en droit, licencié en philosophie, licencié en théologie, membre (de 2002 à 2011) de la Commission fédérale belge de contrôle et d'évaluation de la loi relative à l'euthanasie.

Le législateur belge n'a pas voulu que le contrôle et l'évaluation de l'application de la loi belge relative à l'euthanasie soient exercés directement par les instances judiciaires. Ainsi la loi du 28 mai 2002 relative à l'euthanasie prévoit-elle, dans son chapitre V, qu'entre le champ de l'application de la loi et le pouvoir judiciaire, une commission soit créée, qui agisse comme instance intermédiaire. J'exposerai ici la composition, la mission, les activités de cette commission ainsi que les résultats auxquels elle est parvenue. Je formulerai également quelques remarques critiques.

\section{LE CHAPITRE V DE LA LOI RELATIVE À L'EUTHANASIE}

\section{LA COMMISSION DE CONTRÔLE ET D'ÉVALUATION: CRÉATION \\ ET COMPOSITION}

L'article $6, \S 1^{\text {er }}$ de la loi relative à l'euthanasie prévoit qu'il soit institué «une Commission fédérale de contrôle et d'évaluation de l'application de la présente loi ». Cette commission a été créée en septembre 2002. Conformément aux exigences de la loi (art. 6, §2), elle se compose de seize membres, désignés sur la base de leurs connaissances et de leur expérience dans les matières qui relèvent de sa compétence. Huit membres sont docteurs en médecine, dont quatre au moins professeurs dans une université belge. Quatre membres sont professeurs de droit dans une université belge ou avocats. Quatre membres sont issus des milieux chargés de la problématique des patients atteints d'une maladie incurable. Les membres sont nommés par arrêté royal délibéré en Conseil des ministres, sur une liste double 
présentée par le Sénat, pour un terme renouvelable de quatre ans. La composition de la commission respecte une parité linguistique (néerlandais-français) et assure une représentation pluraliste. Ces deux caractéristiques sont en effet décisives en Belgique: ce pays est trilingue, aux deux langues dominantes précitées s'ajoute de façon minoritaire l'allemand; il est aussi marqué par la présence de deux familles idéologiques dominantes, l'une d'inspiration chrétienne, l'autre d'inspiration laïque.

\section{LES MISSIONS DE LA COMMISSION}

ET LE DOCUMENT DE DÉCLARATION

Cette commission a pour mission de contrôler et d'évaluer les déclarations que les médecins ayant pratiqué une euthanasie doivent lui remettre dans les quatre jours ouvrables. Ces déclarations doivent être remises sous la forme d'un document d'enregistrement qui a été établi par la commission conformément aux articles 5 et 7 de la loi. Ce document est composé de deux volets: I et II. Le volet I contient des données personnelles concernant le patient, le médecin traitant et les personnes, notamment les médecins qui ont été consultés ${ }^{1}$. Il est confidentiel et doit être transmis par le médecin traitant à la commission. Il ne peut être consulté qu'après une décision de celle-ci et ne peut pas servir de base à sa mission d'évaluation (art. 7).

Le volet II est également confidentiel et contient des données concernant:

1. le sexe, les date et lieu de naissance du patient;

2. la date, le lieu et l'heure du décès;

3. la mention de l'affection accidentelle ou pathologique grave et incurable dont souffrait le patient;

4. la nature de la souffrance qui était constante et insupportable ;

5. les raisons pour lesquelles cette souffrance a été qualifiée d'inapaisable;

6. les éléments qui ont permis de s'assurer que la demande a été formulée de manière volontaire, réfléchie et répétée et sans pression extérieure;

7. si l'on pouvait estimer que le décès aurait lieu à brève échéance;

8. s'il existe une déclaration de volonté ;

9. la procédure suivie par le médecin;

10. la qualification du ou des médecins consultés, l'avis et les dates de ces consultations;

11. la qualité des personnes consultés par le médecin, et les dates de ces consultations:

12. la manière dont l'euthanasie a été effectuée et les moyens utilisés (art. 7)2
Puisque la loi fait la distinction entre une euthanasie sur «demande» du patient conscient (art. 3) et une euthanasie de patients inconscients sur base d'une «déclaration anticipée» (art. 4), la commission a ajouté aux douze points du volet II prévus par la loi, sept autres points (13-19) réservés aux euthanasies pratiquées en vertu d'une déclaration anticipée:

13. la date de cette déclaration;

14. le fait que l'inconscience du/de la patient(e) était irréversible ;

15. le fait qu'un médecin indépendant ait été consulté, sa qualification, son avis quant à l'irréversibilité de la situation médicale du/de la patient(e), la date de la consultation;

16. le fait qu'un entretien ait eu lieu avec la ou les personne(s) de confiance éventuellement désignée(s) dans la déclaration anticipée, avec l'équipe soignante, avec les proches du/de la patient(e) désignés par la personne de confiance;

17. le fait que d'autres personnes ou instances aient été consultées, leur qualité et la date de ces consultations;

18. la manière dont l'euthanasie a été pratiquée et les moyens utilisés;

19. les informations complémentaires que le médecin souhaiterait donner (art. 4).

\section{LE TRAVAIL DE LA COMMISSION}

Pendant les réunions qui ont lieu mensuellement, la commission examine les documents d'enregistrement. Elle vérifie, sur la base du volet II du document, si l'euthanasie a été effectuée selon les conditions et la procédure prévues par la loi. En cas de doute, elle peut décider, à la majorité simple, de lever l'anonymat. Elle prend alors connaissance du premier volet. Elle peut demander au médecin traitant de lui communiquer tous les éléments du dossier médical relatifs à l'euthanasie, qui lui paraissent nécessaires.

Lorsque, par décision prise à la majorité des deux tiers, la commission estime que les conditions légales n'ont pas été respectées, elle envoie le dossier au procureur du roi du lieu du décès du patient (art. 8). Jusqu'à présent, de tels envois n'ont pas eu lieu. À vrai dire, en créant la commission, le législateur avait l'intention d'éviter autant que possible de tels envois qui pourraient conduire à des procédures judiciaires. En pratique, la commission demande très souvent des informations supplémentaires avant d'approuver la déclaration. Afin d'instruire et d'informer mieux les médecins à ce sujet, la commission a publié une brochure à l'intention du corps médical contenant des interprétations de quelques notions ou paragraphes de la loi (Commission, Brochure).

\section{LES RAPPORTS BISANNUELS}

DES TRAVAUX DE LA COMMISSION

Tous les deux ans, la commission doit établir, à l'intention des Chambres législatives, un rapport contenant:

a. des statistiques basées sur les informations recueillies dans le volet II des documents d'enregistrement,

b. une description et une évaluation de l'application de la loi relative à l'euthanasie,

c. le cas échéant, des recommandations susceptibles de déboucher sur une initiative législative et/ou d'autres mesures concernant l'exécution de la loi (art. 9).

Un rapport des travaux de la commission a été envoyé aux Chambres législatives en 2004, 2006, 2008 et 2010 (Commission, Rapports).

\section{QUELQUES DONNÉES STATISTIQUES}

\section{APPROCHE GÉNÉRALE}

La commission a commencé ses travaux dès octobre 2002. Le nombre de documents d'enregistrement reçus pendant les premiers quinze mois des travaux couverts par le premier rapport était de 259 , ce qui correspond à une moyenne annuelle de 207,2. Dans les années 2004-2005, le nombre de documents était de 742 (349 pour 2004, 393 pour 2005); en 2006, il était de 429, en 2007, de 495; en 2008, il était de 704, en 2009, de 822 et, en 2010, de 952.

En 2009, le pourcentage des euthanasies "prévisibles à brève échéance» (art. 3, $\S 1$ et 2 ) était de $92 \%$ contre $8 \%$ pour les euthanasies "non prévisibles à brève échéance» $(\operatorname{art} .3, \S 3 \text { et } 4)^{3}$; celui des euthanasies pratiquées sur demande (art. 3) était de 97\%, contre 3\% par déclaration anticipée (art. 4).

Le pourcentage de décès par euthanasies déclarées était de $0,2 \%$ en 2003 et de 0,7 \% en 2009 par rapport à la totalité des décès dans notre pays pendant chacune de ces années.

\section{EUTHANASIES DÉCLARÉES \\ ET EUTHANASIES RÉELLEMENT \\ PRATIQUÉES}

La commission n'est pas en mesure d'évaluer la proportion du nombre d'euthanasies déclarées par rapport au nombre d'euthanasies réellement pratiquées. Une enquête menée avant 2002 dans le Nord du pays estimait que le pourcentage d'euthanasies, alors clandestines, s'élevait à $0,3 \%$ de l'ensemble des décès d'une année, tandis qu'en 2010 on peut estimer que le 
pourcentage des euthanasies déclarées s'élève à $0,7 \%$ de la totalité des décès de l'année (Deliens et al., 2000). On peut dès lors présumer que le nombre des euthanasies clandestines est actuellement très bas en région flamande. Cependant, l'emploi, en fin de vie, de traitements médicaux qui peuvent écourter celle-ci ou y mettre fin (arrêter les traitements, administrer des doses élevées de morphine, pratiquer une sédation) continue à créer des ambiguiités, et ce d'autant plus que, dans ces cas, rien ne doit être déclaré à la commission.

À vrai dire, quoique les trois traitements précités soient reconnus comme des actes "normaux» de l'art médical, ils ont un statut que je qualifierais d'«un peu spécial». Puisqu'ils sont pratiqués très souvent en fin de vie, ces traitements étaient appropriés, avant 2002, à ce qu'on appelait, dans la terminologie de cette époque «euthanasie passive ou indirecte». Mais, déjà alors, ils étaient soupçonnés d'être employés clandestinement comme des moyens pour effectuer des euthanasies actives. Aujourd'hui, ils font partie des traitements quotidiennement administrés dans le cadre des soins palliatifs et ne peuvent plus être considérés comme des formes d'euthanasie. En effet, en Belgique comme aux Pays-Bas, le terme « euthanasie» est légalement défini comme «l'acte, pratiqué par un tiers, qui met intentionnellement fin à la vie d'une personne à la demande de celle-ci» (art. 2). Or, les termes «euthanasie passive» ou «euthanasie indirecte» n'impliquaient pas - du moins théoriquement - qu'on avait l'intention de mettre fin à la vie d'une personne. En ce qui concerne la dernière, on savait qu'indirectement, on pouvait écourter la vie, mais l'intention principale était d'apaiser les douleurs, et la mort pouvait être considérée comme un effet qui n'était pas voulu: elle n'était ni le but, ni le moyen d'apaiser les douleurs. En ce qui concerne la première, on ne voulait pas allonger une agonie - qui était inévitable - en sachant que continuer à traiter n'aurait eu aucun effet thérapeutique. Le terme «passif» suggérait qu'on voulait «laisser mourir » le malade, « laisser la nature faire son travail», comme on le disait alors. Cela n'empêche que le terme "passif » est ambigu puisqu'on décidait tout de même d'arrêter les traitements, et «décider» est, dans ce cas-ci, un acte moralement très important.

Depuis deux ans, on constate d'ailleurs, aux Pays-Bas comme en Belgique, une augmentation des sédations palliatives qui aboutissent au décès du patient. Chez nos voisins hollandais, des médecins, mais aussi des patients, avouent préférer la sédation à l'euthanasie ${ }^{4}$. Cette pratique est évidemment ambiguë puisque, comme je le signalais plus haut, de tels décès ne doivent pas être déclarés auprès de la commission comme des euthanasies. Toutefois, si à la demande du patient, un médecin avait l'intention de pratiquer une euthanasie de cette manière, cette sédation entrerait dans le champ d'application de la loi, et devrait donc être déclarée en bonne et due forme à la commission ${ }^{5}$. Si au contraire le patient ne l'a pas demandée, une telle sédation doit être considérée comme un délit. C'est par là que pourrait s'instaurer actuellement la pratique d'euthanasies clandestines telles que celles qui étaient réalisées avant la loi de 2002.

\section{DIFFÉRENCES ENTRE LE NORD \\ ET LE SUD DU PAYS}

Dès le début, la langue de rédaction des documents a permis de constater une grande divergence entre le Nord du pays (la Flandre et une minorité de Bruxelles) et le Sud (la Wallonie, qui inclut la communauté germanophone, et la majorité de Bruxelles). Constamment, le pourcentage des documents néerlandophones atteint de 80 à $83 \%$ de la totalité des déclarations soumises à la commission, tandis que le pourcentage des documents francophones ne s'élève que de 17 à $20 \%$. Cette disproportion s'explique par le fait que les médecins francophones tiennent davantage à leur liberté thérapeutique et acceptent mal l'intervention des pouvoirs publics dans la relation médecin-patient. De plus, dès 2003, des initiatives ont été prises au Nord du pays pour informer et instruire les médecins sur les manières d'effectuer une euthanasie; des équipes de médecins ayant reçu une formation en vue de donner conseil ou d'aider à effectuer l'euthanasie sont ainsi à la disposition de leurs collègues. Enfin, on ne peut pas oublier que, dans la région flamande du pays - où l'on parle la même langue qu'aux Pays-Bas -, le débat public concernant l'euthanasie a été fort influencé et encouragé par l'approbation, dix ans avant la loi belge, d'une loi relative à l'euthanasie aux Pays-Bas.

Cette disproportion entre le Nord et le Sud du pays se reflète aussi dans les médias. Au Nord, les journaux, les hebdomadaires et la télévision présentent régulièrement des cas d'euthanasies qui ont été réalisées. Il s'agit presque toujours de cas-limites controversés. Les médecins concernés les présentent comme des testcase dans les médias et espèrent par là relancer le débat ${ }^{6}$.

De plus, un petit groupe de médecins généralistes flamands plaide pour que l'euthanasie soit considérée comme acte médical «normal» et demandent l'abrogation de la loi. La plupart d'entre eux sont spécialisés dans les soins palliatifs et cette pratique les a conduits à estimer qu'un changement d'optique est nécessaire: selon eux, l'euthanasie doit être conçue de manière nouvelle. Jadis, estiment-ils, l'acte d'euthanasie était en contradiction avec la finalité de la médecine: en effet, celle-ci doit servir la vie et la santé des personnes. Et ils poursuivent leur argumentation: or, la finalité des soins palliatifs est toute différente de celle de la médecine; les praticiens en soins palliatifs s'occupent des malades qui ne peuvent plus guérir; leur mission est d'apaiser les souffrances par tous les moyens médicalement disponibles. Ainsi, dans l'optique des soins palliatifs, l'euthanasie est-elle le moyen ultime d'apaisement des souffrances dans le cas où le malade la demande à cause de ses souffrances qui sont devenues insupportables et inapaisables. Ces médecins concluent: l'euthanasie est donc conforme à la logique et à la finalité des soins palliatifs. Par conséquent, étant un acte médical normal, elle ne doit pas être légitimée par une loi. Toutefois, il est important de signaler que, dans la législation actuelle, le patient peut refuser les actes médicaux ou y consentir, mais il ne peut pas les demander. Dans le contexte de la proposition de ces médecins, le droit de demander l'euthanasie disparaîtrait.

Les idées de ces médecins sont rejetées par la grande majorité du corps médical. Il est vrai que l'intention de l'euthanasie est in casu "d'apaiser la souffrance insupportable et inapaisable », mais il faut reconnaître que cet acte a des suites qu'on ne peut qualifier de «secondaires ou peu importantes ». Les médecins savent que ces collègues sont bien intentionnés, «idéalistes ». Cependant, ils n'ont pas l'air de tenir compte du fait que leurs opinions bien intentionnées peuvent conduire à des abus.

Au Sud du pays, le silence prédomine. En se rappelant que le pourcentage des déclarations francophones n'atteint qu'à peine les $20 \%$ de la totalité des déclarations faites pour l'ensemble du pays, on peut supposer que les pratiques clandestines d'avant 2002 se poursuivent au Sud. Tout cela démontre que légiférer à propos des traitements médicaux en fin de vie n'est pas facile.

\section{CONTRÔLE ET ÉVALUATION}

\section{LA DOUBLE MISSION}

\section{DE LA COMMISSION ET SES OBJECTIFS}

Le contrôle des dossiers par la commission a pour but de vérifier si toutes les données requises sont correctement rapportées, c'est-à-dire celles qui concernent l'identité du patient, les date et 
lieu de décès, l'échéance prévisible de celui-ci, la date de la demande écrite ou de la déclaration anticipée, la confirmation que l'inconscience du/de la patient(e) est irréversible, la conformité de la procédure suivie avec la loi, l'avis des médecins consultants, la manière dont a été pratiquée l'euthanasie et les produits utilisés.

L'évaluation, qui se rapporte surtout aux points 3 à 7 du document d'enregistrement $^{7}$, a pour but de juger si les euthanasies sont effectuées conformément à la loi.

\section{LE TRAVAIL D'ÉVALUATION DE LA COMMISSION}

\section{NATURE DE L'AFFECTION}

ET «SITUATION MÉDICALE SANS ISSUE »

Au point $3 \mathrm{du}$ document d'enregistrement, le médecin traitant doit donner un diagnostic précis de la nature de l'affection accidentelle ou pathologique grave et incurable dont souffrait le patient et en raison de laquelle ce dernier se trouvait dans une situation médicale sans issue.

Cette évaluation ne pose guère de problèmes, puisqu'il s'agit toujours d'affections graves: des cancers $(79 \%)$; des affections neuromusculaires évolutives (6\%) (sclérose latérale-amyotrophe, sclérose-multiple); des cas de maladie de Parkinson, ou encore la coexistence de plusieurs pathologies incurables (15\%). Le caractère incurable est déterminant pour qu'on puisse parler d'une situation médicale sans issue. Cela veut dire que «la situation médicale sans issue» posée comme condition légale défend au médecin d'effectuer une euthanasie aussi longtemps qu'un traitement curatif est disponible.

SOUFFRANCE CONSTANTE

\section{ET INSUPPORTABLE}

L'article 7 de la loi prévoit que dans le point 4 du document d'enregistrement, le médecin traitant doive faire mention de la nature (physique ou psychique) de la souffrance constante et insupportable. La commission demande en définitive plus de précisions: en effet, au point 4 de ce document d'enregistrement, les médecins doivent donner, en outre, une description de la souffrance. Ainsi, sont mentionnées comme souffrances de nature physique, la cachexie, les douleurs, la dyspnée, la dysphagie, l'épuisement, les hémorragies, l'obstruction digestive, les paralysies, les plaies, les transfusions répétées, et comme souffrances psychiques, la dépendance, la perte de tout espoir à guérir, la perte de dignité ou de qualité de vie. Plusieurs types de souffrances physiques et psychiques sont mentionnées simultanément. De plus, certains cas examinés par la commission ont fait apparaître que l'estimation du caractère insupportable et inapaisable de la souffrance devait tenir compte de l'âge du patient. La notion de "souffrance psychique » a fait l'objet d'échanges de vues au sein de la commission. Certains membres
Celle-ci, n'étant pas nécessairement une souffrance psychique ou physique (art. 3, $\S 1^{\mathrm{er}}$, al. 4), ne peut être déterminante dans l'évaluation de la commission. En effet, n'oublions pas que ce qui est déterminant

IL CONVIENT D'INSISTER SUR L'IMPORTANCE DE CE PRINCIPE D’ÉQUILIBRE, REQUIS PAR LA LOI, ENTRE DEUX ÉLÉMENTS: D'UNE PART, L’ESTIMATION PAR LE MÉDECIN TRAITANT — CONFIRMÉE PAR UN AUTRE MÉDECIN CONSULTÉ — DES ÉLÉMENTS MÉDICAUX (AFFECTION ACCIDENTELLE OU PATHOLOGIQUE GRAVE ET INCURABLE DONT SOUFFRAIT LE PATIENT, CRÉANT UNE SITUATION SANS ISSUE, ET UNE SOUFFRANCE INAPAISABLE); D'AUTRE PART, SON ESTIMATION DU CARACTÈRE INSUPPORTABLE DE SA SOUFFRANCE. CE PRINCIPE D'ÉQUILIBRE OUVRE LE DROIT DE DEMANDER L'EUTHANASIE, MAIS PAS DE FAIRE VALOIR UN DROIT À L'EUTHANASIE.

ont estimé qu'une évolution dramatique future ne pouvait être qualifiée hic et nunc de souffrance psychique insupportable et inapaisable conformément aux termes de la loi relative à l'euthanasie. Toutefois, ce point de vue n'a pas été celui de la majorité de la commission.

\section{SOUFFRANCE INAPAISABLE}

Le point 5 couvre les raisons pour lesquelles cette souffrance a été qualifiée d' «inapaisable ». Les points 4 et 5 s'avèrent ainsi complémentaires. En effet, estimer le caractère insupportable d'une souffrance est en grande partie d'ordre subjectif et dépend de la personnalité du patient; ainsi, c'est son opinion qui va prévaloir dans le point 4 . Au contraire, dans le point 5 , c'est d'abord le médecin traitant qui juge des raisons pour lesquelles la souffrance est qualifiée d' «inapaisable».

Dans la complémentarité des points 4 et 5 , on reconnaît une idée centrale de la loi, l'équilibre entre la volonté du patient d'une part, le jugement médical d'autre part. Très souvent, ces deux points 4 et 5 donnent lieu à des échanges de vue dans la commission. Il n'y a pas lieu de s'en étonner puisque les termes dont il faut juger au point 4 - la ou les souffrances, et leur caractère insupportable - sont susceptibles de plusieurs interprétations. On constate d'ailleurs une tendance à élargir de plus en plus le sens de ces termes: ainsi fait-on désormais souvent mention de "souffrance existentielle». tout d'abord, c'est la constatation d'une affection accidentelle ou pathologique grave et incurable (art. 3, $\S 1^{\mathrm{er}}$, al. 3). S'il en était autrement, on risquerait de créer un déséquilibre entre les critères objectifs et subjectifs, à l'avantage de l'autonomie du patient. Ce risque est d'autant plus grand qu'il faut tenir compte du fait que, même si le médecin estime la souffrance objectivement apaisable (point 5 du document d'enregistrement), le patient a le droit de refuser un traitement de la souffrance, par exemple lorsque ce traitement comporte des effets secondaires ou des modalités d'application qu'il juge insupportables. La commission a toutefois estimé que, dans ces cas, une discussion approfondie entre le médecin et le patient est nécessaire.

\section{DEMANDE VOLONTAIRE, RÉFLÉCHIE,} RÉPÉTÉE ET SANS PRESSION EXTÉRIEURE

Le point $6 \mathrm{du}$ document d'enregistrement consiste à rendre compte des éléments qui ont permis de s'assurer que la demande du patient a été formulée de manière volontaire, réfléchie et répétée et sans pression extérieure. Les réponses sont habituellement satisfaisantes.

Revenons à ce qui a été dit ci-dessus, à savoir qu'«une idée centrale» de la loi belge relative à l'euthanasie est l'équilibre entre la volonté ou l'autonomie du patient d'une part, le jugement du médecin d'autre part. Si, dans la section sur la souffrance inapaisable, on relevait l'équilibre requis 
entre ce qui est demandé dans les points 4 et 5 du volet II, cet équilibre se manifeste plus clairement encore entre ce que la commission doit évaluer au point 6 et ce dont elle doit juger au point $3^{8}$.

Il convient d'insister sur l'importance de ce principe d'équilibre, requis par la loi, entre deux éléments : d'une part, l'estimation par le médecin traitant - confirmée par un autre médecin consulté - des éléments médicaux (affection accidentelle ou pathologique grave et incurable dont souffrait le patient, créant une situation sans issue, et une souffrance inapaisable); d'autre part, l'autonomie du patient (sa demande réfléchie et répétée; son estimation du caractère insupportable de sa souffrance). Ce principe d'équilibre ouvre le droit de demander l'euthanasie, mais pas de faire valoir un droit à l'euthanasie. Ceci n'a pas été - et n'est pas encore - évident, ni pour l'opinion publique, ni pour tous les membres au sein de la commission ${ }^{9}$.

\section{PROXIMITÉ DU DÉCÈS}

Quant au point 7 du volet II, la commission considère que l'échéance du décès doit être estimée "non brève » si la mort n'est pas attendue dans les mois qui viennent (ce qui vaut en pratique pour les affections non évolutives ou très lentement évolutives). Quand le décès est attendu dans les jours, semaines ou mois qui viennent, il peut être considéré comme prévisible à brève échéance.

$* \star *$

En conclusion de cet aperçu du travail d'évaluation de la commission, on peut dire que les interprétations données par celle-ci aux réponses des médecins dans les points 3, 4, 5 et 6 du volet II, constituent en quelque sorte une base pour une «jurisprudence» dans le futur.

\section{QUELQUES PROBLÈMES DE QUALIFICATION}

\section{TRAITEMENTS MÉDICAUX EN FIN DE VIE} ET EUTHANASIE

Dans la section Euthanasies déclarées et euthanasies réellement pratiquées, j'ai déjà relevé l'ambiguïté pouvant résulter de l'emploi des traitements médicaux qui, en fin de vie, peuvent écourter ou terminer celle-ci. Rappelons qu'avant l'approbation, en 2002, de la loi relative à l'euthanasie, il $\mathrm{y}$ eut des propositions visant à incorporer une régulation de ces traitements médicaux en fin de vie dans la loi relative à l'euthanasie. Mais ces propositions n'ont pas été acceptées en raison du fait que le législateur belge n'a pas voulu dépénaliser complètement l'euthanasie ${ }^{10}$. Néanmoins, le milieu médical ressentait la nécessité d'une régulation dans ce domaine. Dans plusieurs cliniques, des procédures ont été rédigées, qui doivent être suivies lors de l'administration des traitements médicaux en fin de vie. Aux Pays-Bas, l'Association royale néerlandaise des médecins a établi un protocole contenant les procédures à suivre dans ces circonstances, et la loi a rendu ce protocole obligatoire pour tout le pays. Ceci constitue un bel exemple d'autorégulation professionnelle légalement approuvée.

Pour sa part, la commission belge a rencontré quelques problèmes de qualification juridique à la suite de l'emploi des traitements médicaux précités: ces situations devaient-elles être considérées ou non comme des euthanasies? J'en parle dans les paragraphes suivants.

\section{SUICIDE MÉDICALEMENT ASSISTÉ}

Plusieurs documents d'enregistrement auprès de la commission belge indiquaient que la technique utilisée pour l'euthanasie avait consisté à obtenir l'inconscience, non par injection I.V. de Thiopental ou de produits similaires, mais par administration d'une dose létale d'un barbiturique en potion que le patient avait avalé lui-même. Dans la majorité de ces cas, le décès s'était produit rapidement sans autre intervention et dans certains cas, un paralysant neuromusculaire avait été injecté après la perte de conscience.

Une telle manière d'agir peut être qualifiée de "suicide médicalement assisté». La commission a cependant considéré qu'elle est autorisée par la loi pour autant que les conditions et les procédures légales autorisant l'euthanasie aient été respectées et que l'acte se soit déroulé sous la responsabilité du médecin présent et prêt à intervenir. En effet, la loi n'impose pas la manière dont l'euthanasie doit être pratiquée.

\section{ARRÊT D'UN TRAITEMENT VITAL}

Dans un autre cas, l'arrêt d'un traitement vital pratiqué à la demande du patient atteint d'une affection incurable avait entraîné le décès en quelques jours sans que des substances létales aient été administrées. La commission a estimé qu'un tel arrêt de traitement n'entre pas dans le cadre de l'euthanasie et n'impose pas de déclaration obligatoire pour autant qu'il soit l'unique cause du décès et que seuls des soins de confort aient été utilisés entre le moment de l'arrêt thérapeutique et le décès. Cependant, si, à la suite de l'arrêt d'un traitement vital, la situation entraîne une demande claire d'euthanasie de la part du patient, la procédure légale doit alors être suivie.

\section{ADMINISTRATION DE MORPHINE}

Les documents d'enregistrement ne mentionnent que rarement une euthanasie pratiquée par l'administration de morphine. L'administration de fortes doses de morphine étant fréquente en fin de vie pour apaiser les souffrances, la commission interprète l'absence de déclaration dans ces cas comme le fait que les médecins voient dans cette manière d'agir un traitement de la souffrance et non une euthanasie, même si elle a pu accélérer le décès. Dans ce cas, il s'agit effectivement d'une attitude médicale normale. Cependant, si l'administration de doses élevées de morphiniques et de sédatifs n'est pas justifiée par le besoin d'apaiser les souffrances du patient mais fait suite à une demande du patient de mettre fin à sa vie, il s'agit bien d'une euthanasie au sens légal du terme: celle-ci est alors soumise aux conditions et procédures légales, y compris l'obligation de déclaration. Rappelons que la loi n'impose pas de technique médicale particulière pour la pratique de l'euthanasie.

\section{RECOMMANDATIONS}

\section{INFORMATION DES MÉDECINS \\ ET DU PUBLIC, FORMATION \\ DES MÉDECINS}

L'article 9 de la loi relative à l'euthanasie prévoit que, dans les rapports à l'intention des Chambres législatives, la commission présente, le cas échéant, des recommandations susceptibles de déboucher sur une initiative législative et/ou d'autres mesures concernant l'exécution de la présente loi.

Dans ses rapports, la commission insiste sur la nécessité d'un effort d'information, tant vis-à-vis des médecins que des citoyens.

La brochure d'information destinée au corps médical est systématiquement adressée aux médecins dont le volet I du document d'enregistrement a été ouvert, soit pour de simples remarques, soit pour demander des explications complémentaires.

Quant à la brochure d'information destinée au public, outre les clarifications utiles pour ce dernier concernant le champ d'application de la loi, ce document doit attirer l'attention sur l'importance de la déclaration anticipée d'euthanasie dans les cas d'inconscience irréversible où les décisions médicales sont particulièrement difficiles à prendre. Plusieurs initiatives ont été prises dans ce sens par les mutualités, par l'Association pour le droit de mourir dans la dignité (ADMD) et d'autres associations qui s'occupent particulièrement des patients atteints d'une maladie incurable. 
La recommandation la plus importante de la commission concerne la formation des médecins. La commission estime que le curriculum des études médicales devrait comporter une formation permettant d'affronter les problèmes que pose la gestion de la fin de vie, y compris les soins palliatifs et la mise en œuvre correcte d'une euthanasie. Elle rappelle que plus de 40\% des citoyens sont assistés médicalement en fin de vie.

\section{CONCLUSIONS}

\section{QUALITÉ DES TRAVAUX}

DE LA COMMISSION

La commission a été instituée en 2002. J'estime qu'elle peut présenter un bilan positif de ses travaux. Sa composition est garante d'un examen professionnel des dossiers. Je considère que le contrôle et l'évaluation obligatoires de toutes les euthanasies pratiquées est plus efficace, plus direct et constructif que des poursuites judiciaires.

Les membres de la commission ont le temps de prendre connaissance des documents d'enregistrement avant les réunions, et - rappelons-le - la commission ne peut délibérer valablement que si les deux tiers de ses membres sont présents. Ni les différences idéologiques des membres, ni les différences fort importantes d'approches des dossiers en fonction des disciplines professionnelles - par les médecins et les personnes issues des milieux chargés des soins de patients en fin de vie d'une part, par les juristes d'autre part - n'empêchent que les débats se déroulent toujours en toute sérénité et sont marqués par une bienveillance respectueuse à l'égard de tous les membres.

\section{QUALITÉ DES DÉCLARATIONS}

\section{SOUMISES À LA COMMISSION}

$\mathrm{Au}$ cours des années 2008-2009, 85\% des déclarations ont été acceptées d'emblée; dans $15 \%$ des dossiers, la commission a décidé la levée de l'anonymat par ouverture du volet I.

Cette ouverture a été, dans $4 \%$ des déclarations, justifiée par la volonté de la commission de faire remarquer au médecin des imperfections dans ses réponses ou des erreurs d'interprétation concernant les procédures suivies qui ne mettaient pas en cause le respect des conditions légales. Dans ces cas, aucune réponse du médecin n'était sollicitée.

Dans 11\% des déclarations, l'ouverture du volet I a été destinée à obtenir des renseignements complémentaires souhaités par la commission à propos d'un ou de plusieurs points du document, mal, insuffisamment ou non complétés.

\section{AUTRES LOIS COMPLÉMENTAIRES}

Rappelons qu'en Belgique, une loi relative aux droits du patient ainsi qu'une loi relative aux soins palliatifs ont été promulguées au cours de l'année 2002, c'est-à-dire l'année où la loi relative à l'euthanasie a été décrétée. Il aurait d'ailleurs été inconcevable que la pratique d'euthanasie soit légalement autorisée sans une législation parallèle relative à la protection des droits du patient et à l'institution des soins palliatifs. Dans les dossiers examinés par la commission, on constate très souvent qu'une euthanasie est effectuée après que le patient eut bénéficié pendant un certain temps de soins palliatifs, ou, qu'ayant le droit de refuser les soins palliatifs, il a demandé immédiatement l'euthanasie.

\section{DE NOUVELLES INITIATIVES LÉGISLATIVES?}

Les membres de la commission soutiennent des opinions diverses à propos de la loi actuelle relative à l'euthanasie: certains veulent l'élargir, d'autres préfèrent le statu quo, quelques-uns veulent la restreindre. Mais la commission refuse de se prononcer à cet égard. Dans ses quatre rapports, elle confirme toutefois que, n'ayant pas de compétence pour donner un avis politique à propos du contenu de la loi, l'application de celle-ci, dans sa forme actuelle, ne donne pas lieu à des difficultés majeures ou à des abus qui nécessiteraient de nouvelles initiatives législatives.

\section{Bibliographie}

CLAESSENS, Patricia, Johan MENTEN, Paul SCHOTMANS, Bert BROECKAERT (2008), "Palliative sedation: a review of the research literature», Journal of Pain and Symptom Management, vol. 36, $\mathrm{n}^{\circ}$ 3, p. 310333.

COMMISSION FÉDÉRALE DE CONTRÔLE ET D'ÉVALUATION DE L'EUTHANASIE, Brochure à l'intention $d u$ corps médical, accessible à l'adresse : <http:// www.health.fgov.be/euthanasie>.

COMMISSION FÉDÉRALE DE CONTRÔLE ET D'ÉVALUATION DE L'EUTHANASIE, "Rapport aux Chambres Législatives », 2004, 2006, 2008 et 2010, accessibles à l'adresse: <http://www.health. fgov.be/euthanasie $>$.

DELIENS, Luc et al. (2000), «End-of-life decisions in medical practice in Flanders, Belgium : a nation-wide survey », The Lancet, 356, p. 1806-1811.

HERREMANS, Jacqueline (2011/2012), «Mourir dans la dignité. La loi belge relative à l'euthanasie, une réponse légale ", Frontières, vol. $24, \mathrm{n}^{\text {os }} 1-2$, p. 73-80.
LOIS BELGES (pour voir les modifications apportées, consulter: <http://www.cass. be>) :

Loi du 28 mai 2002 relative à l'euthanasie, Moniteur belge, 22 juin 2002.

Loi du 14 juin 2002 relative aux soins palliatifs, Moniteur belge, 26 octobre 2002.

Loi du 22 août 2002 relative aux droits du patient, Moniteur belge, 26 septembre 2002.

\section{Notes}

1. Ces données personnelles concernent les nom, prénoms et domicile du patient; les nom, prénoms, numéro d'enregistrement à l'INAMI (Institut national d'assurances maladie et invalidité) et domicile du médecin traitant, du(des) médecin(s) qui a (ont) été consulté(s) concernant la demande d'euthanasie; les nom, prénoms, domicile et qualité de toutes les personnes consultées par le médecin traitant, ainsi que les dates de ces consultations; s'il existait une déclaration anticipée et qu'elle désignait une ou plusieurs personnes de confiance, les nom et prénoms de la(les) personne(s) de confiance qui est(sont) intervenue(s).

2. Tous les points du volet II doivent être remplis par le médecin traitant, et non par le médecin à qui le médecin traitant aurait demandé éventuellement d'effectuer l'euthanasie. Pour de plus amples explications concernant les notions mentionnées dans le volet II, voir dans ce numéro de Frontières l'article de Jacqueline Herremans.

3. En ce qui concerne la différence entre euthanasie "prévisible» ou "non prévisible» à brève échéance, voir notre titre: Proximité $d u$ décès ainsi que l'article de Jacqueline Herremans dans ce numéro de Frontières.

4. Rappelons que durant les dernières années, les techniques à pratiquer la sédation palliative ont été perfectionnées très rapidement. Dans plusieurs centres universitaires, les médecins-spécialistes qui travaillent dans les unités de soins palliatifs ont acquis une grande maîtrise de ce traitement et atteignent une très haute précision pour ce qui concerne la durée voulue et souhaitable de la sédation. Voir Patricia Claessens, Johan Menten, Paul Schotmans, Bert Broeckaert (2008), «Palliative Sedation: a Review of the Research Literature", Journal of Pain and Symptom Management, vol. 36, $\mathrm{n}^{\circ}$ 3, p. 310-333.

5. Voir nos titres: Traitements médicaux en fin de vie et euthanasie, Suicide médicalement assisté, Arrêt d'un traitement vital.

6. Le premier de ces «reality-programmes» a été présenté quelques semaines après l'approbation de la loi. C'était vraiment une "leçon-modèle»: l'émission montrait en effet comment le malade s'était préparé à l'effectuation de l'euthanasie; ses entrevues avec le médecin traitant, et avec la famille; la préparation de la chambre où l'euthanasie serait effectuée; la réception d'adieux avec la famille et quelques amis. Entretemps, on expliquait que, dans ce cas, toutes les conditions légales avaient été remplies. Enfin, le programme s'achevait avec une interview de la fille qui avait assisté aux derniers moments de son père. 
Ces dernières années, les médias (presse, télévision, radio) ont relayé plusieurs cas très controversés.

1/ Tout d'abord, celui d'Hugo Claus, poète et romancier flamand, notoire défenseur depuis longtemps de l'autonomie de la personne, pas seulement dans le sens d' "autodétermination» mais aussi d' « autodisposition ». Il affirmait : «je veux décider moi-même du moment où je meurs». On avait constaté chez lui les premiers symptômes de la maladie d'Alzheimer. Pour lui, la perspective de subir des dégradations ultérieures physiques et surtout mentales (confusion, ne plus trouver les mots exacts pour s'exprimer) était vécue comme une souffrance insupportable: c'était à ses yeux une raison suffisante pour demander l'euthanasie.

2/ Une dame nonagénaire, légèrement handicapée, était lasse de vivre plus longtemps. Elle demandait l'euthanasie. Plusieurs médecins-généralistes avaient refusé d'y consentir. Finalement, elle a trouvé un médecin qui l'a «aidée».

3/ Une dame, déjà légèrement démente, demandait l'euthanasie car elle voulait "prendre congé de ses proches et mourir consciemment». La perspective de mourir dans l'inconscience, après avoir passé une longue période dans un état comateux, était vécue par elle comme une souffrance insupportable.

Remarquons que dans les cas 1 / et 3/, il s'agit de la perspective d'une évolution dramatique future qui est vécue hic et nunc comme insupportable. C'est ce qu'on pourrait appeler «euthanasie anticipée ou préventive» (voir notre titre: Souffrance constante et insupportable, in fine).
7. Voir notre titre: Les missions de la commission et le document de déclaration, $2^{\mathrm{e}} \S$.

8. Voir notre titre: Nature de l'affection et "situation médicale sans issue».

9. Le droit à l'euthanasie supposerait que l'euthanasie soit tout à fait dépénalisée. Or, ce n'est pas le cas. La loi de 2002 fait certes partie du code pénal. Cependant, dans la mesure où elle n'autorise l'euthanasie que sous les conditions qu'elle prévoit, on peut en conclure qu'elle ne dépénalise que partiellement l'euthanasie. En voici quelques exemples. Le $1^{\mathrm{er}}$ alinéa de l'article 3 pose que: "Le médecin qui pratique une euthanasie ne commet pas d'infraction s'il s'est assuré que $[. .]$.$» . De même l'article 4, \S 2$ réitère la restriction: "un médecin qui pratique une euthanasie, à la suite d'une déclaration anticipée, [...], ne commet pas d'infraction s'il constate que le patient [...]». Enfin, l'article 8, $\S 3$ s'exprime aussi en ce sens : «Lorsque, par décision prise à la majorité des deux tiers, la commission estime que les conditions prévues par la présente loi n'ont pas été respectées, elle envoie le dossier au Procureur du Roi du lieu du décès du patient. ».

Il faut d'ailleurs souligner que, si le patient avait un droit à l'euthanasie, ce droit aurait pu être légalement réglé dans la loi du 22 août 2002 relative aux droits du patient.

10.Voir notre titre: Demande volontaire, réfléchie, répétée et sans pression extérieure, ainsi que la note 9. 